\title{
ANGIOMA CAVERNOSO DO MEATO ACÚSTICO INTERNO ENVOLVENDO O COMPLEXO VII E VIII NERVOS CRANIANOS
}

\section{Relato de caso}

\author{
Roberto Leal Silveira', Gustavo Cardoso de Andrade2, Nilson Pinheiro Júnior ${ }^{3}$, \\ José Eymard Homem Pittella4, Vinícius Cotta Barbosa ${ }^{5}$
}

\begin{abstract}
RESUMO - Relata-se o caso de homem de 21 anos, leucoderma, portador de angioma cavernoso do meato acústico interno direito envolvendo o complexo VII e VIII nervos cranianos tratado cirurgicamente. Apenas 18 casos de angiomas cavernosos dessa localização foram relatados na literatura. São comentados os aspectos clínicos, o diagnóstico diferencial e o tratamento.
\end{abstract}

PALAVRAS-CHAVE: angioma, cavernoma, meato acústico interno, neurinoma do acústico.

\begin{abstract}
Cavemus hemangioma of the internal auditory canal encasing the VII and VIII cranial nerve complex: case report

ABSTRACT - We report the surgically treated case of a 21-year-old caucasian male harboring a cavernous hemangioma of the right internal auditory canal encasing the seventh and eighth cranial nerves complex. Only 18 cases of cavernous hemangiomas of this location have been previously reported. The clinical features, the differential diagnosis and the treatment are discussed.
\end{abstract}

KEY WORDS: angioma, cavernoma, internal auditory canal, acoustic neuroma.

Angiomas cavernosos, malformação cavernosa ou cavernomas são malformações vasculares compactas angiograficamente ocultas com incidência de 0,3 a $0,5 \%$, correspondendo a 10 a $20 \%$ das malformações vasculares ${ }^{1-3}$. Ocorrem de forma esporádica ou familial. Quando familial, a ocorrência de lesões múltiplas é mais comum². Localizam-se habitualmente nos hemisférios cerebrais, podendo também ocorrer no compartimento infratentorial e medula espinhal ${ }^{1-6}$. Angiomas cavernosos dos nervos cranianos são raros, apenas 44 casos descritos, 18 dos quais envolvendo o complexo VII e VIII nervos' ${ }^{1}$. A Iocalização no interior do meato acústico interno justifica sua inclusão entre as lesões incomuns a serem consideradas no diagnóstico diferencial dos neurinomas do acústico.

Descrevemos o caso de um paciente jovem com angioma cavernoso do meato acústico interno envolvendo o complexo VII-VIII nervos cranianos, desde seu diagnóstico até o tratamento cirúrgico.

\section{CASO}

Homem de 21 anos, leucoderma, com relato de perda progressiva de acuidade auditiva à direita há cerca de oito anos, com piora acentuada da audição em novembro de 2003, quando foi encaminhado ao Serviço de Neurocirurgia do Hospital Madre Teresa. Sem quaisquer outros déficits ao exame neurológico. Audiometria de julho de 2003 revelava perda média de 50 decibéis, com discriminação vocal de $70 \%$. Novo exame realizado em novembro de 2003 já não apresentava discriminação auditiva. Ressonância magnética (RM) mostrou lesão expansiva intrameatal no lado direito medindo aproximadamente um centímetro, isointensa em relação ao parênquima cerebral nas ponderações T1 e T2, sem captação significativa do meio de contraste. A janela óssea da tomografia computadorizada

Neurocenter, Serviço de Neurocirurgia do Hospital Madre Teresa Belo Horizonte, MG, Brasil: 'Mestre e Doutor em Neurocirurgia, Chefe do Serviço de Neurocirurgia do Hospital Madre Teresa; ${ }^{2}$ Residente em Neurocirurgia do Hospital Madre Teresa; ${ }^{3}$ Neurocirurgião do Hospital Madre Teresa; ${ }^{4}$ Professor Titular do Departamento de Anatomia Patológica e Medicina Legal da Faculdade de Medicina da Universidade Federal de Minas Gerais; ${ }^{5}$ Otoneurologista.

Recebido 31 Maio 2004, recebido na forma final 12 Agosto 2004. Aceito 2 Outubro 2004. 


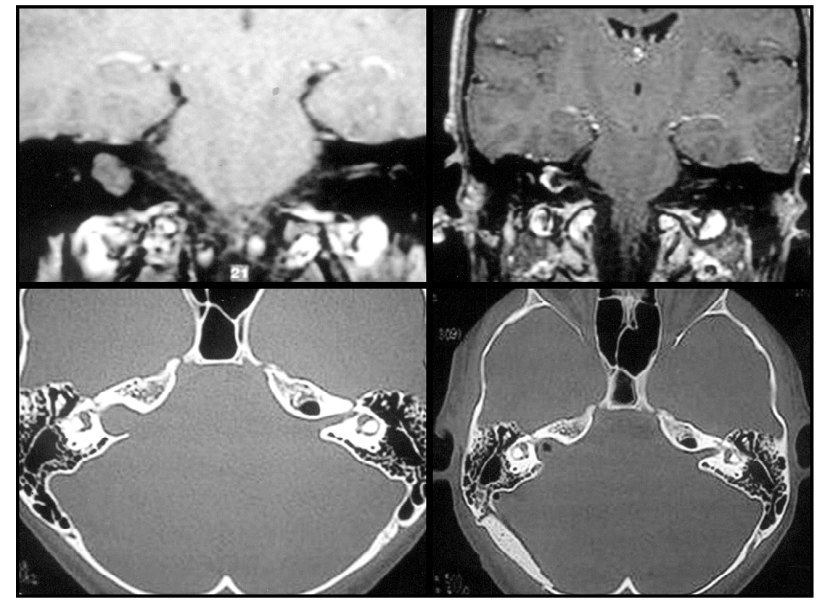

Fig 1 A e B. Imagens por RM (T1 pós-gadolíneo) e TC (janela óssea) pré-operatórias, demonstrando, respectivamente, lesão expansiva intrameatal com grande alargamento do meato acústico interno direito, porém sem destruição importante poro acústico interno. C e D: Imagens por RM (T1 pós-gadolíneo) e TC (janela óssea) pós-operatório, observando-se, respectivamente, ressecção total da lesão e abertura ampla da parede posterior do meato acústico interno direito (acesso transmeatal).

(TC) caracterizou destruição óssea importante do meato acústico interno ipsilateral (Fig $1 \mathrm{a}, 1 \mathrm{~b}$ ).

Indicado tratamento cirúrgico por acesso retrossigmóideo transmeatal direito em posição semi-sentada. Após abertura dural e afastamento medial do cerebelo, notou-se coloração atípica dos nervos vestibulares na entrada do poro acústico interno, possivelmente devendose à impregnação por hemossiderina. Durante a abertura do meato acústico, identificou-se lesão vascular em íntima associação com o VII e VIII nervos, aderida sobretudo ao nervo coclear, que foi totalmente ressecada usandose técnica microcirúrgica (Fig 2).
O período pós-operatório transcorreu sem intercorrências. Nesse período realizou RM e TC de alta resolução para ossos temporais que revelaram exérese total da lesão (Fig 1c, 1d). O paciente recebeu alta hospitalar no quinto dia de pós-operatório apresentando anacusia total à direita, sem paresia facial (grau I de House e Brackmann) e sem outros déficits neurológicos.

O exame histopatológico revelou lesão vascular benigna formada pela proliferação de vasos sanguíneos justapostos de luz ampla e parede fibrosa, espessa, freqüentemente hialinizada, revestida por camada simples de endotélio plano, alterações essas características de hemangioma cavernoso (cavernoma) (Fig 3).

O presente relato foi autorizado pela Comissão de Ética do Hospital Madre Teresa e obteve-se consentimento informado do paciente.

\section{DISCUSSÃO}

Os angiomas cavernosos do complexo VII-VIII nervos tendem a se manifestar através da diminuição da acuidade auditiva, podendo ter evolução insidiosa, rapidamente progressiva ou súbita. Paresia facial também pode ocorrer e, segundo alguns autores, a presença de paresia facial ou déficit auditivo acentuado nos portadores de pequenas lesões intracanaliculares é sugestiva de lesão vascular, sendo dado importante no diagnóstico diferencial dos schwannomas do vestibular, ainda que não haja como diferenciá-los a partir de dados clínicos ${ }^{1,4,7,8}$.

Os cavernomas habitualmente apresentam-se heterogêneos à RM, apresentando áreas isointensas e hiperintensas em relação ao parênquima encefálico nas ponderações T1 e T2, além de captar pouco contraste. $O$ diagnóstico diferencial é feito sobretudo com o schwannoma do vestibular, que é hiperin-

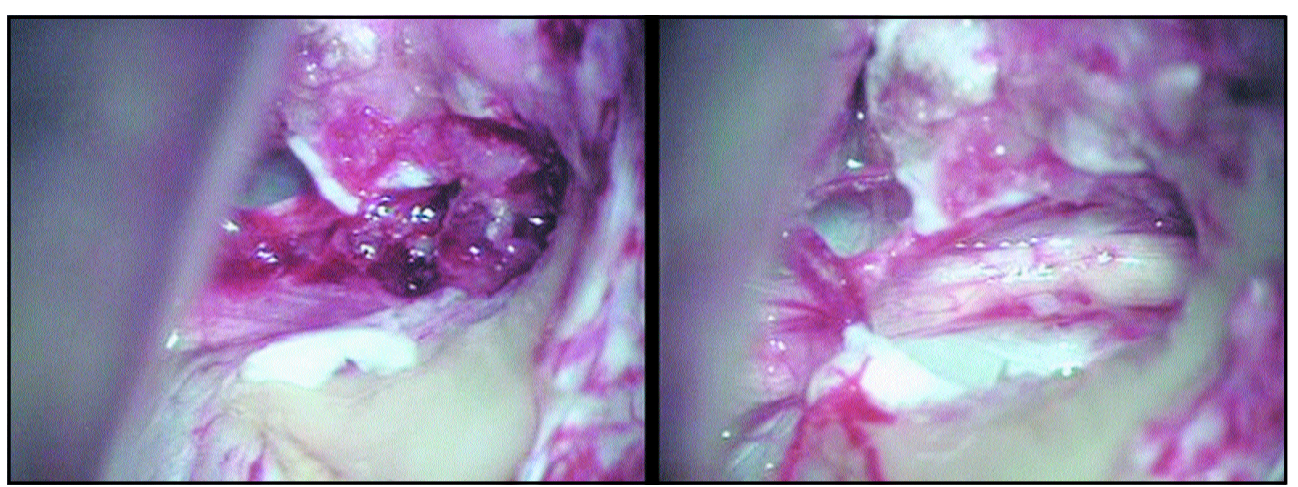

Fig 2 A. Exposição trans-operatória após abertura da parede posterior do meato acústico interno. Nota-se lesão no interior do meato acometendo o complexo VIIIVIII nervos cranianos. B: Vista transoperatória do mesmo paciente após ressecção completa da lesão. Observa-se a preservação das estruturas neuro va saulares no interior do meato acústico interno: nervos facial e coclear anteriormente, nervos vestibulares superior e inferiormente e ramo da AICA (artéria cerebelar antero-inferior). 


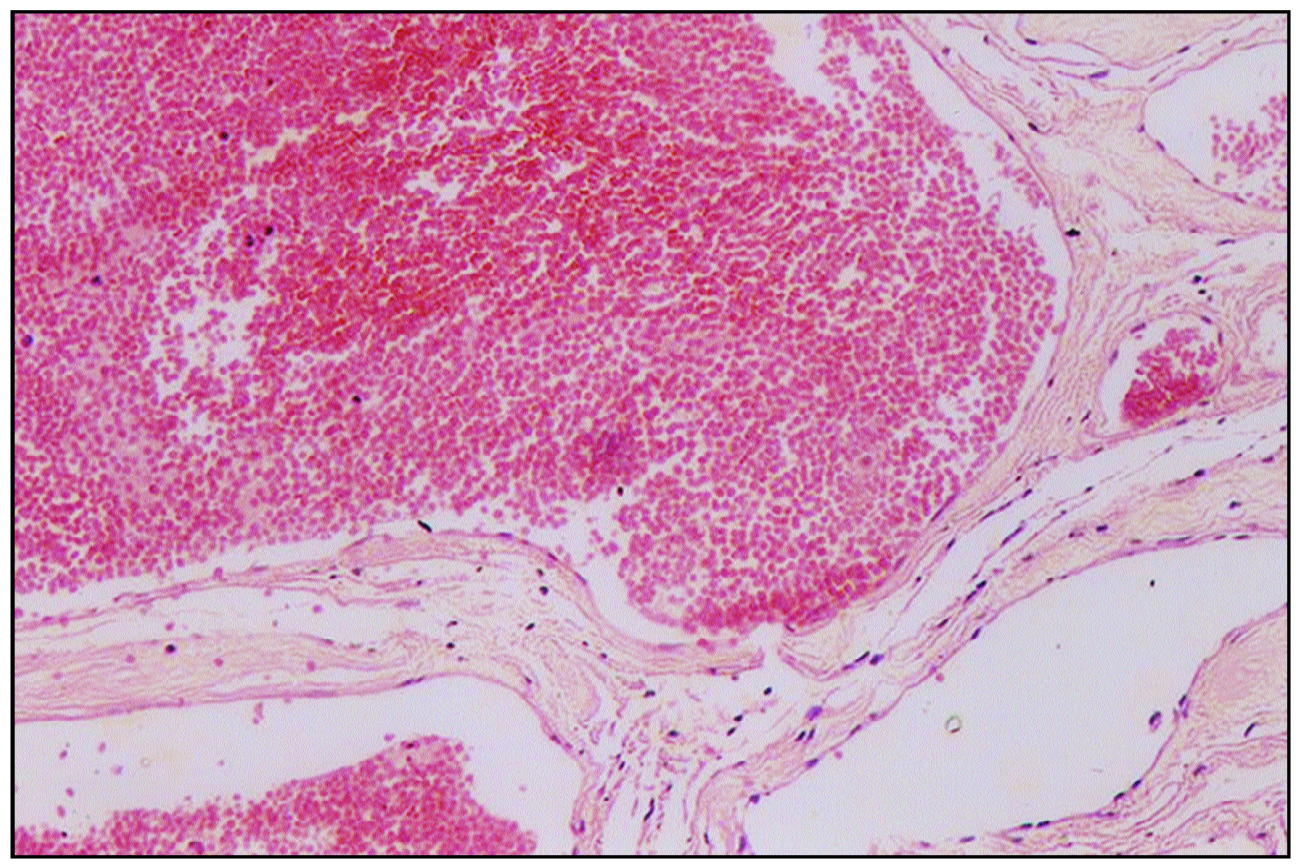

Fig 3. Angioma cavernoso. Vasos sanguíneos justapostos, de luz ampla e parede fibrosa revestida por camada simples de endotélio plano. Hematoxilina-Eosina, aumento original 180X.

tenso em T2, hipointenso em T1, com captação importante do contraste paramagnético. No presente caso, a apresentação isointensa em T1 e T2, sem captação do meio de contraste, dificultou o diagnóstico pré-operatório.

Essas lesões vasculares podem apresentar calcificações no interior do meato acústico interno e destruição deste, melhor visibilizados na janela para osso da $\mathrm{TC}^{4,5,7,8}$. O cavernoma desse presente relato ocasionou alargamento pronunciado do interior do meato acústico interno, sem alargar o poro acústico interno, diferenciando-se dos schwannomas do vestibular, que tendem a alargar o poro e em seguida o meato acústico interno.

A ressecção microcirúrgica total é o tratamento de escolha dos cavernomas do complexo VII-VIII nervos, haja vista a importância dessa região anatômica e a possibilidade do aparecimento de déficits neurológicos importantes ${ }^{1-4,6,7}$. Ressecção subtotal pode levar à recorrência da lesão'.

Em conclusão, os angiomas cavernosos do complexo VII-VIII nervos são lesões raras que devem ser consideradas no diagnóstico diferencial das lesões do ângulo pontocerebelar e meato acústico interno, sendo a ressecção microcirúrgica total seu tratamento de escolha.

\section{REFERÊNCIAS}

1. Deshmukh VR, Albuquerque FC, Zabramski JM, Spetzler RF. Surgical management of cavernous malformations involving the cranial nerves. Neurosurgery 2003;53:352-357.

2. Meneses MS, Dallolmo VC, Kondageski C, Ramina R, Hunhevicz S, Pedrozo AA. Ciru rgia estereotáxica guiada para angiomas cavernosos. Arq Neuropsiquiatr 2000;58:71-75.

3. Matias-Guiu X, Alejo M, Sole T, Ferrer I, Noboa R, Bartumeus F. Cavernous angiomas of the cranial nerves: report of two cases. J Neurosurg 1990;73:620-622.

4. Babu R, Ransohoff J, Cohen N, Zagzag D. Cavernous angiomas of the internal auditory canal: a case report and review of the literature. Acta Neurochir (Wien) 1994;129:100-104.

5. Kim M, Rowed DW, Cheung G, Ang LC. Cavernous malformation presenting as an extra-axial cerebellopontine angle mass: case report. Neurosurgery 1997;40:187-190.

6. Iplikçioglu AC, Benli K, Bertan V, Ruacan S. Cystic cavernous hemangioma of the cerebellopontine angle: case report. Neurosurgery 1986; 19:641-642.

7. Pappas DG, Schneiderman TS, Brackmann DE, Simpson SC, ChandraSekar B, Sofferman RA. Cavernous hemangiomas of the internal auditory canal. Otolaryngol Head Neck Surg 1989;101:27-32.

8. Bricolo A, Micheli E, Gambin R, Alessandrini F, Iuzzolino P. Cavernous malformation of the internal auditory canal.J Neurosurg Sci 1995;39:153-158. 